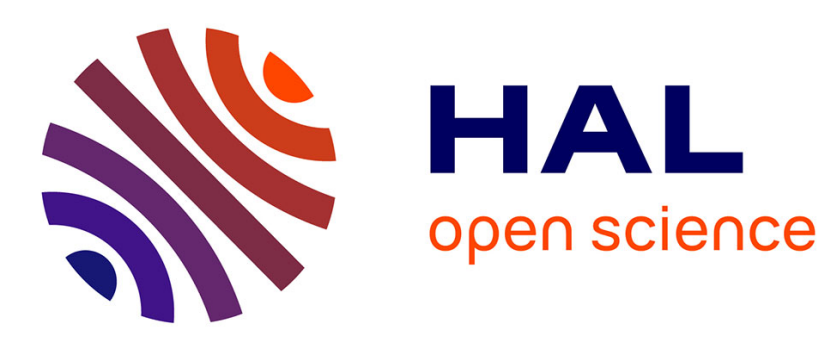

\title{
The effect of applied stresses on the equilibrium moisture content in polymers
}

Katell Derrien, Pierre Gilormini

\section{To cite this version:}

Katell Derrien, Pierre Gilormini. The effect of applied stresses on the equilibrium moisture content in polymers. Scripta Materialia, 2007, 56 (4), p. 297-299. 10.1016/j.scriptamat.2006.10.001 . hal00755493

\section{HAL Id: hal-00755493 \\ https://hal.science/hal-00755493}

Submitted on 21 Nov 2012

HAL is a multi-disciplinary open access archive for the deposit and dissemination of scientific research documents, whether they are published or not. The documents may come from teaching and research institutions in France or abroad, or from public or private research centers.
L'archive ouverte pluridisciplinaire HAL, est destinée au dépôt et à la diffusion de documents scientifiques de niveau recherche, publiés ou non, émanant des établissements d'enseignement et de recherche français ou étrangers, des laboratoires publics ou privés. 


\title{
The effect of applied stresses on the equilibrium moisture content in polymers
}

\author{
K. Derrien ${ }^{1}$, P. Gilormini \\ Laboratoire d'Ingénierie des Matériaux (CNRS UMR 8006), \\ ENSAM, 151 Boulevard de l'Hôpital, 75013 Paris, France
}

\begin{abstract}
The relation between saturation moisture content in a polymer and applied stresses is derived as a function of the coefficient of moisture expansion. The model predictions are compared favourably with experimental data taken from the literature.
\end{abstract}

Keywords: Polymers; Diffusion; Thermodynamics; Moisture.

\section{Introduction}

The absorption of moisture by polymers may induce swelling (see for instance Weitsman [1] for a review on the effects of fluids on polymer-matrix composites). Since swelling is a strain, it interacts with the stresses that are applied to the polymer. More specifically, an isotropic swelling (as considered below) interacts with the mean stress, i.e. the hydrostatic pressure, applied. It is the purpose of this paper to analyze the effect of applied stresses on the amount of moisture that a polymer can absorb. An elastic behaviour is assumed for the polymer, and only the equilibrium moisture content is considered, leaving the influence of stresses on the diffusion process to further papers. A typical application of the proposed model is the moisture absorption capacity of composites [2]: the reinforcements do not absorb moisture and restrain the swelling of the resin matrix, where stresses are induced and where the moisture content at saturation is lower than in neat resin, consequently.

\footnotetext{
$\overline{1}$ Corresponding author, Tel.: 331442462 74; fax: 331442462 90, E-mail address katell.derrien@paris.ensam.fr (K. Derrien)
} 


\section{Modification of the chemical potential by applied stresses}

Consider an elementary volume $V_{p}$ of a dry polymer. A number of moles $n_{p}$ can be defined in $V_{p}$ from either the number of molecules or the number of repetitive constitutive units (in the case of a cross-linked polymer) that it contains. Water molecules will come into $V_{p}$ in the sequel, so that an open system can be defined from $n_{p}$, the number of polymer moles that remains constant while the volume $V$ of the system is likely to vary, because of moisture-induced swelling, for instance. Temperature $T$ is assumed constant, and a sufficiently small system is considered, such that its temperature and water content are uniform. The latter is defined as the mass of water $M_{w}$ over the mass of polymer $M_{p}$ in the system : $c=M_{w} / M_{p}=\omega_{w} n_{w} /\left(\omega_{p} n_{p}\right)$, where $\omega_{w}$ and $\omega_{p}$ denote the molecular weights of water and of the polymer, respectively, and $n_{w}$ is the number of moles of water in the system. In addition to $c$, the state of the system is defined by the total strain $\varepsilon$ with respect to the reference state where the system contains dry polymer only and no stress is applied. Moisture uptake is supposed to induce an isotropic swelling of the polymer, such that the swelling strain is given by $\varepsilon^{s}=\eta c \boldsymbol{i}$, where $\eta$ denotes the coefficient of moisture expansion, and $\boldsymbol{i}$ the identity second-order tensor. If swelling can develop freely, the volume of the system becomes $(1+3 \eta c) V_{p}$, consequently. The present approach is limited to linear swelling, but it can be extended to the frequently observed case where a swelling threshold is present, for instance.

The system is assumed to be isotropically elastic, with a bulk modulus $k$ and a shear modulus $G$, so that elastic strains $\varepsilon^{\mathrm{e}}=\varepsilon-\varepsilon^{\mathrm{s}}$ are induced if swelling cannot develop freely, with stresses given by $\boldsymbol{\sigma}=k(\operatorname{tr} \boldsymbol{\varepsilon}-3 \eta c) \boldsymbol{i}+2 G \boldsymbol{e}$, where $\operatorname{tr}($.$) denotes the trace of a second-order tensor, and \boldsymbol{e}$ is the deviatoric part of the total strain $\varepsilon$. The Helmholtz free energy of the system can now be written as follows, when water molecules have been added and a total strain has been prescribed:

$$
F(c, \varepsilon)=F_{0}+n_{p} f(c)+V_{p} W,
$$

where $F_{0}$ is the free energy of the dry and stress-free polymer, $f(c)$ is the variation of the free energy due to the addition of water (with zero applied stresses) per mole of dry polymer, and $W$ is the elastic energy per unit volume

$$
W(c, \boldsymbol{\varepsilon})=\frac{1}{2} \boldsymbol{\sigma}: \boldsymbol{\varepsilon}^{\mathrm{e}}=\frac{k}{2}(\operatorname{tr} \boldsymbol{\varepsilon}-3 \eta c)^{2}+G \boldsymbol{e}: \boldsymbol{e} .
$$

By definition, the generalized chemical potential of water in the system is given by the partial derivative of the Helmholtz free energy with respect to the number of moles of water, for a fixed total strain:

$$
\widetilde{\mu}_{w}=\left.\frac{\partial F}{\partial n_{w}}\right|_{\varepsilon}=\left.\frac{\partial F}{\partial c}\right|_{\varepsilon} \frac{\partial c}{\partial n_{w}}=\left.\frac{\omega_{w}}{n_{p} \omega_{p}} \frac{\partial F}{\partial c}\right|_{\varepsilon},
$$


and one obtains from (1) and (2):

$$
\widetilde{\mu}_{w}=\mu_{w}(c)+\frac{3 \omega_{w} \eta}{\rho_{p}} p+\frac{\omega_{w}}{\rho_{p}}\left[\frac{k^{\prime}(c)}{2}(\operatorname{tr} \varepsilon-3 \eta c)^{2}+G^{\prime}(c) \boldsymbol{e}: \boldsymbol{e}\right]
$$

where $\mu_{w}(c)=\left(\omega_{w} / \omega_{p}\right) f^{\prime}(c)$ is the chemical potential of water in the stressfree polymer, $\rho_{p}=n_{p} \omega_{p} / V_{p}$ is the mass density of the dry polymer, and $p=$ $-\frac{1}{3} \operatorname{tr} \sigma=-k \operatorname{tr} \varepsilon^{\mathrm{e}}$ denotes the hydrostatic pressure associated with the applied stresses. The possible variations of the elastic properties with moisture content have been included in (4), where $k^{\prime}(c)$ and $G^{\prime}(c)$ are the derivatives of $k(c)$ and $G(c)$. In the sequel, these variations will be neglected and therefore the following expression of the chemical potential will be used:

$$
\widetilde{\mu}_{w}=\mu_{w}(c)+\frac{3 \omega_{w} \eta}{\rho_{p}} p
$$

Similar expressions, with a chemical potential that depends linearly on the hydrostatic pressure, have been obtained previously, for instance by Weitsman [3] and $\mathrm{Wu}[4]$, but without the molar mass of water appearing explicitly, which may be due to different definitions of the chemical potential used. Similarly, expressions have also been given for diffusion in metals (Larché and Cahn [5], for instance), but the correspondence between $\omega_{w} / \rho_{p}$ and the density of lattice sites used in the latter context is not trivial. In contrast, (5) coincides with an expression obtained by Li et al. [6] when the partial molar volume $\bar{V}_{w}$ of the mobile component (water, in our case) used by these authors is explicited:

$$
\bar{V}_{w}=\frac{\mathrm{d} V}{\mathrm{~d} n_{w}}=\omega_{w} \frac{\mathrm{d} V}{\mathrm{~d} M_{w}}=\omega_{w} \frac{\mathrm{d} V / V_{p}}{\mathrm{~d} M_{w} / M_{p}} \frac{V_{p}}{M_{p}}=\omega_{w} \frac{3 \eta}{\rho_{p}}
$$

using the definition of $\eta$ as the ratio between the relative volume gain and the relative weight gain. An advantage of (5) is that it refers to directly accessible material parameters only.

Since small strains have been assumed, the variation between the initial and final volumes of the system was neglected above. A more elaborate derivation has been proposed by $\mathrm{Wu}$ [4], where finite strain analysis was applied first, before small strain assumption was used. This leads to an additional term in the generalized chemical potential, which had been obtained already by Li et al. [6] and which is similar to the second term in (5) where $p$ would be replaced by the elastic energy $W$. Since $W$ is of the order of $\frac{p}{2 k} p$, for a zero deviatoric strain, for instance, and since $p$ is much smaller than $k$ in practical applications, the additional term can be neglected with respect to the last term in (5). 


\section{Modification of the water content at saturation by applied stresses}

Let now the elementary volume of polymer considered in the previous Section be exposed to a humid environment. Water molecules can be exchanged between the polymer and the surrounding air, and an equilibrium will settle with some stabilized water content $c$ in the polymer. As is well-known in chemical thermodynamics, equilibrium is obtained when the chemical potentials of water in the humid environment $\hat{\mu}_{w}$ and in the polymer are equal, which writes

$$
\hat{\mu}_{w}=\widetilde{\mu}_{w} .
$$

There remains to use suitable expressions for $\hat{\mu}_{w}$ and $\mu_{w}$, since the latter is a part of $\widetilde{\mu}$ in (5). If the humid air is considered as a mixture of perfect gases, the following standard expression can be used:

$$
\hat{\mu}_{w}=\hat{\mu}_{0}+R T \ln \left(\frac{p_{w}}{p_{0}}\right)
$$

where $p_{0}$ is a reference pressure (usually taken equal to 1 bar), and $\hat{\mu}_{0}$ is the corresponding potential. If the activity can be assimilated to the moisture content, the chemical potential of water in the stress-free polymer can be written as:

$$
\mu_{w}=\mu_{0}+R T \ln \left(\frac{c}{c_{0}}\right)
$$

where $c_{0}$ is a reference moisture content and $\mu_{0}$ the corresponding potential. Using (5), the equilibrium condition leads to

$$
\frac{c}{p_{w}}=\frac{c_{0}}{p_{0}} \exp \left(\frac{\hat{\mu}_{0}-\mu_{0}}{R T}-\frac{3 \omega_{w} \eta p}{R T \rho_{p}}\right) \approx \frac{c_{0}}{p_{0}} \exp \left(\frac{\hat{\mu}_{0}-\mu_{0}}{R T}\right)\left(1-\frac{3 \omega_{w} \eta}{R T \rho_{p}} p\right)
$$

assuming that $p$ is much smaller than $R T \rho_{p} /\left(3 \omega_{w} \eta\right)$. The final result can also be written as

$$
c=S p_{w}(1-A \eta p)
$$

with

$$
S=\frac{c_{0}}{p_{0}} \exp \left(\frac{\hat{\mu}_{0}-\mu_{0}}{R T}\right) \quad \text { and } \quad A=\frac{3 \omega_{w}}{R T \rho_{p}} .
$$

If no stress is applied, Henry's law $c=S p_{w}$ is recovered, which is due to the simple expressions that have been used above for the chemical potentials. If stresses are applied, a modified form of Henry's law is obtained, with a solubility $S^{\prime}=S(1-A \eta p)$ that decreases when the applied hydrostatic pressure increases: less water is absorbed if the polymer is compressed (and more if it is depressed).

This analysis extends immediately to polymers that do not obey Henry's law. Assume that the chemical potential of water in the polymer is more complex 
than (9), and that $c / c_{0}$ should be replaced by the ratio of activities $a(c) / a\left(c_{0}\right)$. Instead of (11), the following would have been obtained:

$$
a(c)=\frac{a\left(c_{0}\right)}{c_{0}} S p_{w}(1-A \eta p) .
$$

Reading the (nonlinear) sorption isotherm of the polymer with no applied stress as $p_{w}(c)$, instead of the usual $c\left(p_{w}\right)$ way, would have provided a direct measure of $\frac{c_{0}}{S} \frac{a(c)}{a\left(c_{0}\right)}$. The isotherm with applied stresses would then be given by multiplying all values along the $p_{w}$ axis by a factor of $1 /(1-A \eta p)$, since (13) leads to

$$
p_{w}=\frac{1}{S} \frac{c_{0}}{a\left(c_{0}\right)} \frac{a(c)}{1-A \eta p} .
$$

In other words, wether Henry's law applies or not, the present theory based on a purely elastic behavior of the polymer suggests that the application of stresses modifies the sorption isotherm by a factor of $1 /(1-A \eta p)$ along the vapor pressure axis, where $A$ can be deduced from the polymer mass density, temperature, and the molar mass of the absorbed species. Weitsman [3] had already obtained a linear law for the saturation level in a polymer under stress, but the coefficients were not explicited. Here, the expression of the saturation law is related to material parameters, and it can be compared to experimental observations. This is done below.

Henson and Weitsman [7] exposed coupons of an epoxy resin (Hercules 3502) to an ambient relative humidity of $97 \%$ at a temperature of $40 \mathrm{C}$, with various applied uniaxial stresses. Several replicates were used for each applied stress, which lead to a range of results as shown in Fig. 1. The mass density of the resin $\rho_{p}$ is $1.26 \times 10^{3} \mathrm{~kg} / \mathrm{m}^{3}$ ([8], for instance), and its coefficient of moisture expansion $\eta$ has been measured by Zimmerman et al. [9] as 0.1925. The molar mass of water is $18 \times 10^{-3} \mathrm{~kg} / \mathrm{mol}, R$ is $8.314 \mathrm{~J} / \mathrm{mol} / \mathrm{K}$, and $T$ is $313 \mathrm{~K}$, so that coefficient $A$ is about $16.5 \mathrm{GPa}^{-1}$. It may be noted that the condition used above for replacing the exponential by a linear term is satisfied: $|p| \ll 1 /(A \eta)$, since the pressures applied in [7] varied from 0 to $-7.8 \mathrm{MPa}$. The only value that is taken from Fig. 1 for comparing the model to the experimental results is the equilibrium moisture content at zero applied stress, that is $S p_{w}$, which is $5.2 \%$ in [7]. This gives the level of the straight line shown in Fig. 1, the slope of which is $\mathrm{d} c / \mathrm{d} \sigma=-3 \mathrm{~d} c / \mathrm{d} p=A S p_{w} \eta / 3$. A very good agreement can be observed between the model prediction and the experimental results.

To the authors knowledge, this is the first time a model is compared to the experimental values of [7], since no curve is plotted on the corresponding figure in [3] or [10], for instance, and several comments must be made. First, the usual explanation for the general trend of the variations of the saturation values is based on a viscoelastic behavior of the resin [3]. This leads to a nonlinear law, that may be quadratic [3] and would give a better agreement with experiments than the linear law obtained here. Nevertheless, this 


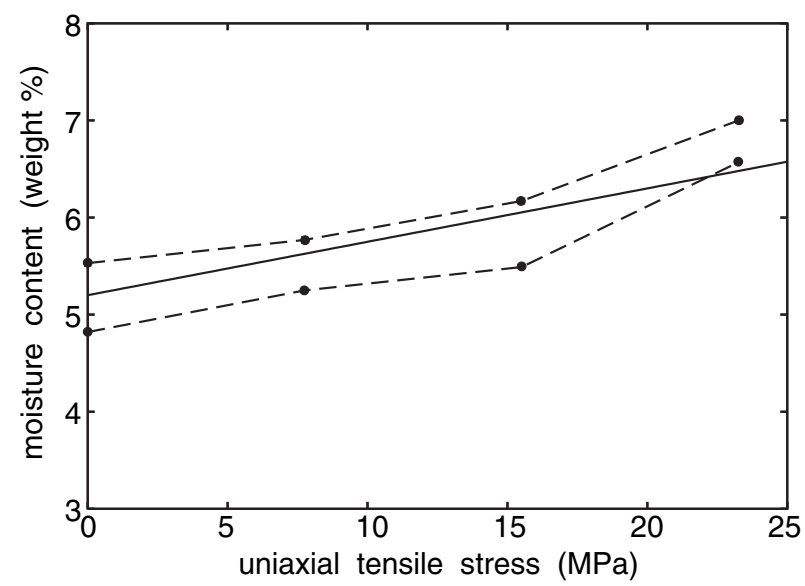

Fig. 1. Moisture content in an epoxy resin under tensile uniaxial stress. The model (unbroken line) is compared to experimental results (bounded by the upper and lower broken lines) obtained by Henson and Weitsman [7] on series of replicate specimens of a Hercules 3502 epoxy at 40C and $97 \%$ RH.

has never been proved quantitatively, probably because the coefficients of the viscoelastic behavior of the resin are not available. Moreover, for very long diffusion times, the elastic ideal behavior considered in the present analysis, which has the advantage of leading to simple developments, may be reasonably pertinent. This is even more justified when the temperature of the tests (40C) is compared to the glass transition temperature of the resin $(240 \mathrm{C}$, as mentioned in [7]). It must also be pointed out that saturation was not completed totally when the weight-gain was measured, as noted by Henson and Weitsman [7], which means that the level of the experimental curves in Fig. 1 should be raised slightly. Since diffusion, and saturation consequently, is likely to be faster when a uniaxial tension is applied, the correction of the results in Fig. 1 would be larger for smaller $\sigma$ values, and the accordance with the proposed model would be improved.

\section{References}

[1] Weitsman YJ. In: Comprehensive Materials, Polymeric Matrix Composites, Talreja R and Manson JAE Eds, Elsevier, 2000, p. 369.

[2] Derrien K, Gilormini P. Submitted for publication.

[3] Weitsman YJ. J. Mech. Phys. Solids 1987; 35:73.

[4] Wu CH. J Mech Phys Solids 2001; 49:1771.

[5] Larché FC, Cahn JW. Acta Metall 1982; 30:1835.

[6] Li JCM, Oriani RA, Darken LS. Z. Phys. Chem. Neue Folge 1966; 49:271. 
[7] Henson MC, Weitsman Y. In: Composites'86: Recent Advances in Japan and the United States, Kawata K, Umekawa S, Kobayashi A, Eds, Tokyo: Japan Society for Composite Materials, 1986, p. 775.

[8] Composite Materials Handbook, vol. 2: Polymer Matrix Composites Materials Properties, Department of Defense, MIL-HDBK-17-2E, 1999.

[9] Zimmerman RS, Adams DF, Walrath DE. Investigation of the Relations Between Neat Resin and Advanced Composite Mechanical Properties, vol. 1: Results, University of Wyoming report UWME-DR-301-101-1, 1984.

[10] Weitsman Y. In: Fatigue of Composite Materials, Reifsnider KL Ed, Elsevier, 1990, p. 385. 